\title{
Two-Dimensional Low Spatial Frequency Laser-Induced Periodic Surface Structuring of Sapphire
}

\author{
Simon Schwarz, Stefan Rung and Ralf Hellmann \\ Applied Laser and Photonics Group, University of Applied Sciences, \\ 63743 Aschaffenburg, Germany \\ E-mail: Simon.Schwarz@h-ab.de
}

\begin{abstract}
We report on the generation of two-dimensional low spatial frequency laser-induced periodic surface structures on transparent material. Inspired by the potential applications in transmission light microscopy, we have successfully structured entire sapphire substrates by using an infrared femtosecond laser having a wavelength of $1030 \mathrm{~nm}$, a pulse duration of $222 \mathrm{fs}$ and a repetition rate of $50 \mathrm{kHz}$, in conjunction with a 2D-galvo scanner with highly uniform low spatial frequency LIPSS. By varying the laser fluence, scanning speed and scanning direction with respect to the polarization of the laser, we optimize the process of 1D and 2D periodic structuring and find scanning orthogonal to the laser polarization with a laser fluence above the ablation threshold is preferential for high quality structuring with a high degree of homogeneity and parallelism and low damage.
\end{abstract}

DOI: $10.2961 /$ jlmn.2017.02.0003

Keywords: laser-induced periodic surface structures, LIPSS, LSFL, transparent materials, sapphire

\section{Introduction}

Laser-induced periodic surface structures (LIPSS) are topic of research since their first observation on semiconductors in 1965 [1]. These periodic structures, typically, arise upon polarized laser irradiation of solids with the fluence being near the materials ablation threshold [2-5]. Two types of LIPSS are distinguished, defined by the relation between their periodicity $\Lambda$ and the applied laser wavelength $\lambda$. Low spatial frequency LIPSS (LSFL) are characterized by having a periodicity $\Lambda_{\mathrm{LSFL}} \approx \lambda$, whereas high spatial frequency LIPSS (HSFL) have $\Lambda_{\mathrm{HSFL}}<<\lambda$ [4-6]. The origin of LSFL has been described by Emmony et al. [7] by an interference of the electromagnetic field of the used laser and a surface scattered wave generated by the incident laser light. Sipe et al. [3] used this approach to develop a mathematical model for the evolution of LSFL, referred to as efficacy factor theory, which is today the generally accepted approach. The surface scattered wave that forms the basis of this theory was later associated to surface plasmon polaritons [5]. For HSFL, however, there is no established theory yet [8-10].

Since, low spatial frequency LIPSS influence the wettability and cell growth of structured surfaces [2, 11-13], LSFL on transparent materials are of particular interest in transmission light microscopy for medical applications. However, the generation of LSFL on transparent materials is, as compared to metals and semiconductors, challenging since initially free charged carriers have to be generated as a prerequisite for the formation of the surface scattered wave. As it has been shown in Ref. 14 and 15, for the generation of LSFL free charged carriers in dielectrics, such as fused silica, are excited by the irradiating laser via multiphoton absorption and avalanche ionization [16].

Punctual HSFL and LSFL on transparent materials, such as fused silica or sapphire, have been demonstrated by several research groups [5, 6, 17-19]. In the case of sapphire, Stoian et al. [17] used and Ti:sapphire laser having a wavelength of $800 \mathrm{~nm}$ with pulse durations of $0.2 \mathrm{ps}$ and $2.8 \mathrm{ps}$. LSFL have been observed for both, $0.2 \mathrm{ps}\left(4.0 \mathrm{~J} / \mathrm{cm}^{2}\right.$,

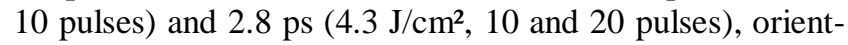
ed perpendicular to the laser polarization with a periodicity corresponding to $\lambda(\approx 0.8 \mu \mathrm{m})$. While HSFL have been observed in the femtosecond regime $(0.2 \mathrm{ps})$ with a periodicity of about $0.2 \mu \mathrm{m}$, LSFL formation is favoured in the picosecond regime (2.8 ps). Ashkenasi et al. [18] also used an Ti:sapphire laser and its second harmonics for the generation of LSFL with 10 pulses on sapphire specimens. Experiments were carried out for $\lambda=790 \mathrm{~nm}$ with pulse durations of $2.3 \mathrm{ps}$ and $1.3 \mathrm{ps}$ and the associated fluence of $6.4 \mathrm{~J} / \mathrm{cm}^{2}$ (2.3 ps) and $3.2 \mathrm{~J} / \mathrm{cm}^{2}(1.3 \mathrm{ps})$, respectively, showing LSFL having a periodicity of $740 \mathrm{~nm} \pm 40 \mathrm{~nm}$. For the second harmonic, pulse durations of 0.2 ps and 1.3 ps were applied with the corresponding fluence of $4.5 \mathrm{~J} / \mathrm{cm}^{2}$ and $3.4 \mathrm{~J} / \mathrm{cm}^{2}$, respectively, revealing to LSFL with periodicities of $360 \mathrm{~nm} \pm 30 \mathrm{~nm}$. For all experiments, the generated LSFL always show an orientation perpendicular to the laser polarization. Qi et al. [19] investigated the femtosecond ablation process of sapphire and found LIPSS, generated with a Ti:sapphire laser $(780 \mathrm{~nm})$, pulse duration of $164 \mathrm{fs}$ and a repetition rate of $1 \mathrm{kHz}$, for a pulse number of 20-50 with a fluence close to the ablation threshold of the material. The LIPSS are oriented perpendicular to the polarization of the laser, having a periodicity of approximately $340 \mathrm{~nm}$ and are therefore rated as being HSFL. In case of HSFL, which are yet not in the focus of this article, also one-dimensional periodic structures along a defined line have been shown on different transparent materials [8, $9,20]$. In a recent publication, we firstly reported the generation of one-dimensional LSFL on wide band gap transparent material, namely fused silica with a specific focus on a rotation of the LSFL orientation in different irradiation regimes [21]. Eberle et al. [22] observed structures appearing as 1D-LSFL on sapphire, though themselves denoted these as ripples. However, opposite to the generally used 
approach to generate LIPSS by a simple and single laser irradiation, the authors in Ref. 22 employed laser irradiation and a subsequent wet etching process to make these nanostructures visible. A similar combined laser/etching approach was reported by Gottmann et al. [23] for structures in fused silica having periodicities as HSFL. Although 1D-LSFL have been explicitly shown on fused silica as a representative for transparent materials, we here show for the first time that it is also possible to generate them on sapphire.

Despite numerous publications of 2D-LSFL on nontransparent materials e.g. metals [11, 24, 25], no literature for 2D-LSFL on dielectrics is available. In this contribution, we report on the generation of 2D-LSFL, enabling spatial coverage of transparent materials, with specific focus on sapphire. The influence of the interplay between laser scanning direction and laser polarization on a distinct occurrence of the LSFL is demonstrated.

\section{Experimental}

An ultrashort pulse laser (Pharos, Light Conversion) having a wavelength of $1030 \mathrm{~nm}$, a repetition rate of $50 \mathrm{kHz}$ and a pulse duration of $222 \mathrm{fs}$ (FWHM) is used for the experimental study. The pulse energy, and thereby the applied fluence is adjusted by an attenuator, while the polarization is controlled with a $\lambda / 2$-plate. The laser beam is deflected by a galvo scanner (RTA AR800 2G+, Newson) and focused on the sample surface with an $f-\theta$-lens having a focal length of $100 \mathrm{~mm}$. The resulting Gaussian focus diameter is $31 \mu \mathrm{m}\left(1 / \mathrm{e}^{2}\right)$ as being measured by a high resolution CCD camera (UI-1490SE-M-GL, IDS).

In this study, sapphire has been chosen as a prominent candidate of transparent materials used in biology and medical research. It comprises good optical properties for microscopic applications, is biocompatible, has a high hardness and is inert [26, 27]. Single-crystalline sapphire substrates with an uncharted cut, having a thickness of $2 \mathrm{~mm}$ and a surface roughness of about $6 \mathrm{~nm}$ are processed (UQG Optics). The surface topography is analysed using a scanning electron microscope (SEM, Phenom ProX, PhenomWorld), an atomic force microscope (AFM, Dimension Icon, Bruker) and a transmitted light microscope (DM6000 $\mathrm{M}$, Leica), respectively. The images taken by the transmitted light microscope and the scanning electron microscope are transferred into Fourier spectra to precisely determine the periodicities of the LSFL and HSFL, respectively [28].

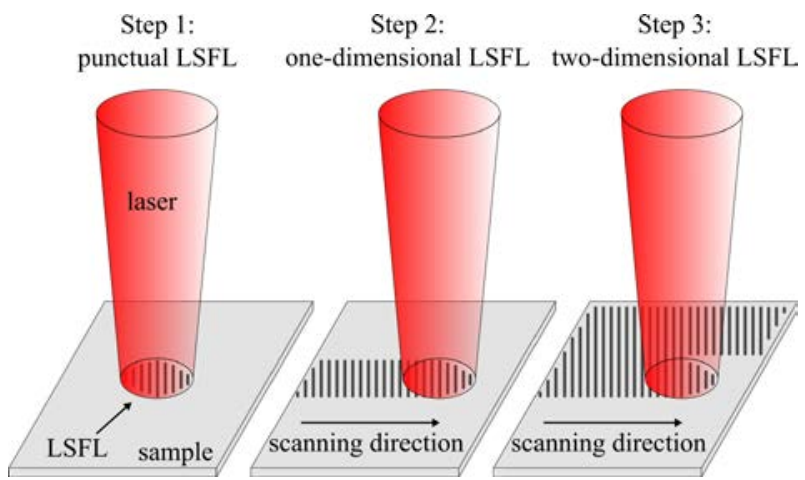

Fig. 1 Schematic illustration of the three step process for structuring a surface with two-dimensional LSFL.
For successfully structuring entire surfaces with LSFL, the generation is split into three steps (cf. figure 1) that are individually optimized. Firstly, the influence of pulse number and fluence on the generation of common punctual LSFL is analysed. Translation of the laser focus by the scanner leads to the generation of one-dimensional LSFL (straight lines). For this second step, the influence of scanning direction, scanning speed and fluence on the 1D-LSFL is determined. By concatenating the one-dimensional structures, 2D-LSFL covering entire surfaces are produced.

\section{Results and Discussion 3.1 Punctual LSFL}

Figure 2 shows SEM images of the evolution of both LSFL and HSFL on sapphire with increasing fluence between $1.63 \mathrm{~J} / \mathrm{cm}^{2}$ and $3.11 \mathrm{~J} / \mathrm{cm}^{2}$ for a fixed number of laser pulses $\mathrm{N}=10$. Apparently, in a low power regime up to about $1.88 \mathrm{~J} / \mathrm{cm}^{2}$, HSFL evolve with an orientation orthogonal to the laser polarization (Fig. 2 a), b)). With increasing fluence, LSFL begin to arise in the centre of the irradiated spot while HSFL remain visible at the rim of the modified area (Fig. 2 c)). This is the typically observed behaviour of punctual LIPSS formation, forming HSFL in a lower fluence regime than LSFL [6, 29, 30]. The HSFL generated with the parameters in figure $2 \mathrm{~b}$ ) reveal a periodicity of $418 \mathrm{~nm} \pm 27 \mathrm{~nm}$, significantly below the wavelength of the laser. The LSFL reveal a periodicity of $924 \pm 6 \mathrm{~nm}$, i.e. slightly below the employed laser wavelength and their orientation is orthogonal to the laser polarization, both being in accordance to previous findings $[17,18]$. It is worthwhile to note that the LSFL formation appears to be not well pronounced, an observation that has earlier been observed for sapphire by Stoian et al. and Ashkenasi et al. $[17,18]$. With a further increase of the fluence to 3.11 $\mathrm{J} / \mathrm{cm}^{2}$, we observe a damage of the sapphire specimen. Ad hoc performed measurements of the ablation threshold for the particular material on hand according to the method of Liu [31] reveal an ablation threshold of $2.97 \mathrm{~J} / \mathrm{cm}^{2}$ (for 10 pulses), confirming that LSFL appear for fluences near the ablation threshold [2-5].
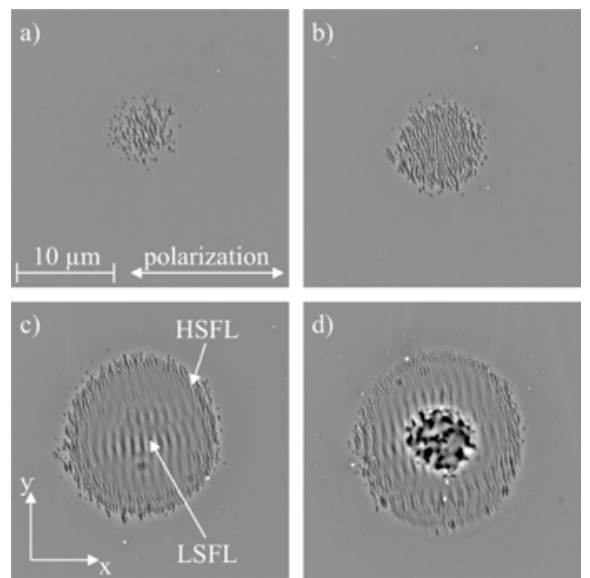

Fig. 2 SEM images of punctual LIPSS on sapphire, generated with $\mathrm{N}=10$ and a) $\Phi=1.63 \mathrm{~J} / \mathrm{cm}^{2}$, b) $\Phi=1.88 \mathrm{~J} / \mathrm{cm}^{2}$, c) $\Phi=2.86 \mathrm{~J} / \mathrm{cm}^{2}$ and d) $\Phi=3.11 \mathrm{~J} / \mathrm{cm}^{2}$ 

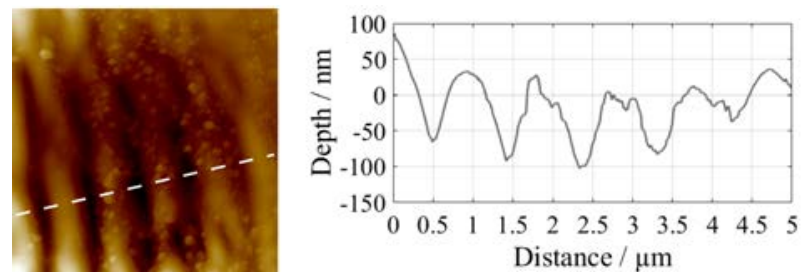

Fig. 3 AFM image $(5 \mu \mathrm{m}$ x $5 \mu \mathrm{m})$ with modulation profile of punctual LSFL on sapphire generated with $\mathrm{N}=10$ and $\Phi=2.86 \mathrm{~J} / \mathrm{cm}^{2}$.

Figure 3 shows an atomic force microscope image with a field of view of $5 \mu \mathrm{m}$ x $5 \mu \mathrm{m}$ (same parameters used for the LIPSS generation as in figure 2 c)), highlighting the associated topography of the LSFL with a modulation depth of about $100 \mathrm{~nm}$ and a periodicity of about $939 \mathrm{~nm}$, respectively.

\subsection{One-dimensional LSFL}

To generate one-dimensional low-spatial frequency LIPSS, the laser beam is scanned across the sapphire sample both parallel and orthogonal to the polarization of the laser. Experiments with varying scanning speeds between $50 \mathrm{~mm} / \mathrm{s}$ and $145 \mathrm{~mm} / \mathrm{s}$ show that qualitatively good onedimensional structures can be generated with a speed of $80 \mathrm{~mm} / \mathrm{s}$ for both directions. Figure 4 shows onedimensional LSFL generated with a scanning direction orthogonal a) and parallel b) to the laser polarization, respectively. For the structures with orthogonal scanning direction (Fig. $4 \mathrm{a}$ )), a fluence of $4.40 \mathrm{~J} / \mathrm{cm}^{2}$ had to be applied to generate clearly resolvable $1 \mathrm{D}$-LSFL. Please note, that opposite for scanning in $\mathrm{x}$-direction $\left(2.47 \mathrm{~J} / \mathrm{cm}^{2}\right)$, this fluence above the ablation threshold results from the reduced spatial overlap of the generated LSFL during the movement of the laser spot in y-direction. Since the LSFL extension is smaller in y-direction (see Fig. 2 c)), for a given pulse-topulse overlap, defined by the scanning speed, a higher fluence is required as to ensure sufficient energy deposition by the Gaussian shaped laser beam profile at the same area (overlap of the spot region with LSFL formation in this direction is about $7 \mu \mathrm{m}$, see Fig. 2 c). The different expansion of the punctual LSFL in the direction perpendicular to the LSFL has already been shown by different authors [3234] and is assigned, to a redistribution of the incident electric field of the laser by the initially induced structures, in turn causing an extension of the electric field distribution perpendicular to the LSFL [32]. This field enhancement in the x-direction can explain the used lower fluence and the

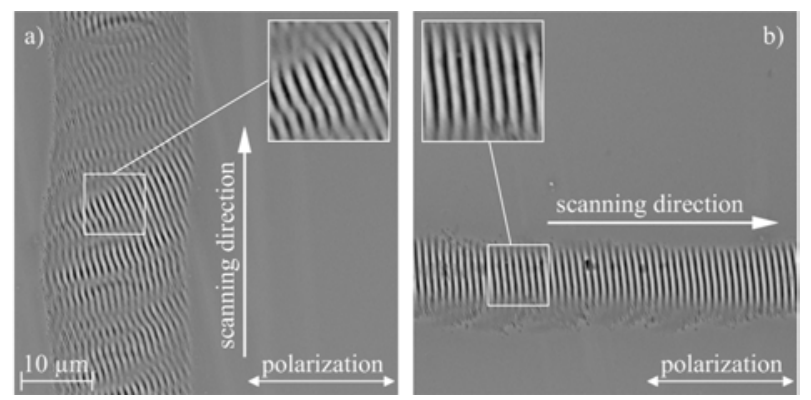

Fig. 4 SEM images of 1D-LSFL on sapphire both with a scanning speed of $80 \mathrm{~mm} / \mathrm{s}$ and a) scanning direction orthogonal and b) parallel to the laser polarization with a) $\Phi=4.40 \mathrm{~J} / \mathrm{cm}^{2}$ and b) $\Phi=2.47 \mathrm{~J} / \mathrm{cm}^{2}$. smaller width of the 1D-LSFL structures for scanning in $\mathrm{x}$ direction (Fig. 4 b)) as compared to scanning in y-direction (Fig. 4 a)). Both the orientation of the 1D-LSFL and the periodicity are almost independent of the scanning direction $\left(\Lambda_{\mathrm{LSFL}}\right.$ is $942 \pm 8 \mathrm{~nm}$ and $951 \pm 7 \mathrm{~nm}$ for orthogonal and parallel scan direction), a behaviour that has also been reported for the orientation of 1D-HSFL on transparent materials [8, 9, 20], or 1D-LSFL on metals [35, 36]. Apparently, the orientation of the LSFL while scanning perpendicular to the laser polarization results not in a perfectly perpendicular orientation of the LSFL towards the laser polarization. A behavior already reported for e.g. 1D-LSFL on chromium and 1D-HSFL on fused silica [8, 37].

The quality of the 1D-LSFL, however, appears to be higher for parallel scanning direction with a higher homogeneity and a high degree of parallelism (Fig. 4 and 5). In addition, for orthogonal scanning direction the applied fluence above the ablation threshold manifests itself in damage on the upper parts of the modulated topography (Fig. 5 a)). This quality difference can be also attributed to the field enhancement discussed before. Fauchet et al. [37] attributed this to the extension of the ripples for scanning perpendicular to the ripple orientation with overlapping pulses. However, in order to ensure the generation of clearly resolvable 2D-LSFL for this scanning direction, a fluence on this level has to be applied. Again, this can be attributed to the smaller extension of the LSFL formation as shown in figure $2 \mathrm{c}$ ).
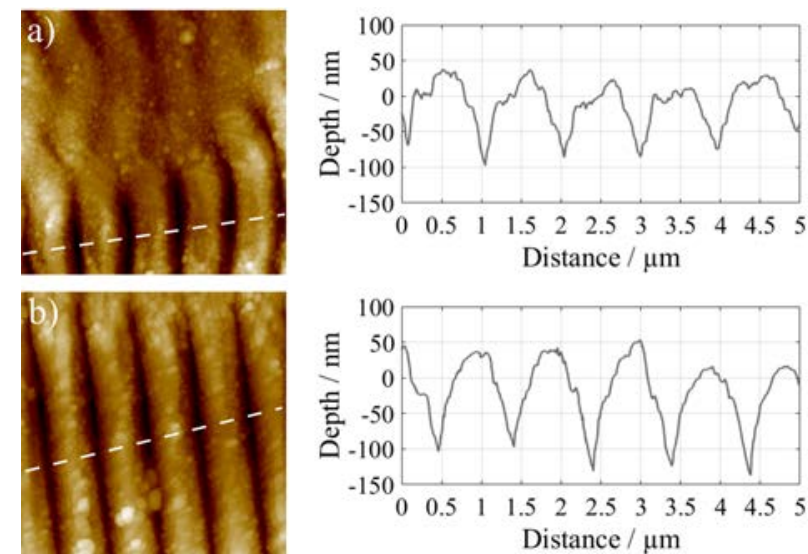

Fig. 5 AFM images $(5 \mu \mathrm{m} \times 5 \mu \mathrm{m})$ with modulation profile of 1D-LSFL on sapphire both with a scanning speed of $80 \mathrm{~mm} / \mathrm{s}$ and a) scanning direction orthogonal and b) parallel to the laser polarization with a) $\Phi=4.40 \mathrm{~J} / \mathrm{cm}^{2}$ and b) $\Phi=2.47 \mathrm{~J} / \mathrm{cm}^{2}$.

\subsection{Two-dimensional LSFL}

The third step illustrated in figure 1 is to transfer the previously generated 1D-LSFL into two-dimensional LSFL by placing one-dimensional LSFL side-by-side with a carefully chosen spacing between the individual 1D-LSFL. Figure 6 a) shows two 1D-LSFL generated by the afore optimized parameters having a line spacing of $20 \mu \mathrm{m}$ with the scanning direction being orthogonal to the laser polarization. As figure 6 a) reveals, the individual 1D-LSFL are partly connected with either LSFL or HSFL without damaging each other. As the 1D-LSFL width is smaller for a scanning direction parallel to the laser polarization, the line spacing for this scanning strategy has to be set to minimum $12 \mu \mathrm{m}$ in 


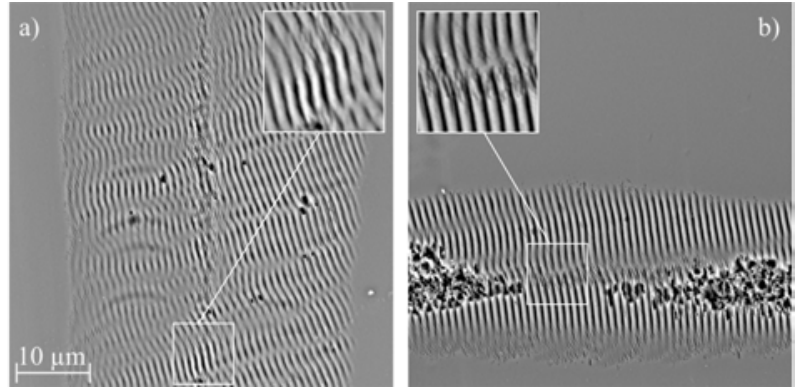

Fig. 6 SEM image of two-dimensional LSFL on sapphire generated with the parameters used in figure 4 and a scanning direction a) orthogonal and b) parallel to laser polarization.

order to achieve 2D-LSFL. Similarly to the results shown in Fig 6 a), the individual 1D-LSFL are partly connected either with LSFL or HSFL. However, we also observe a low quality and partly damage in the overlapping region. Thus, for a high quality 2D-LSFL structuring, an orthogonal orientated scanning direction appears to be preferential. Ruiz de la Cruz et al. [35] reported this qualitatively difference while structuring large areas of chromium. For Cr, LSFL appear perpendicular to the laser polarization (same orientation as for sapphire). In agreement to our experiments, they reported on a higher 2D-LSFL quality for scanning the laser beam parallel to LSFL orientation, linked to the extension of the grating while scanning neighbouring lines.

Figure 7 highlights the successful 2D-LSFL generation on larger regions. Though minor damaged areas remain in the connecting regions between individual 1D-LSFL, the figure clearly shows the homogeneous and continuous surface structuring by highly uniform low spatial frequency LIPSS.

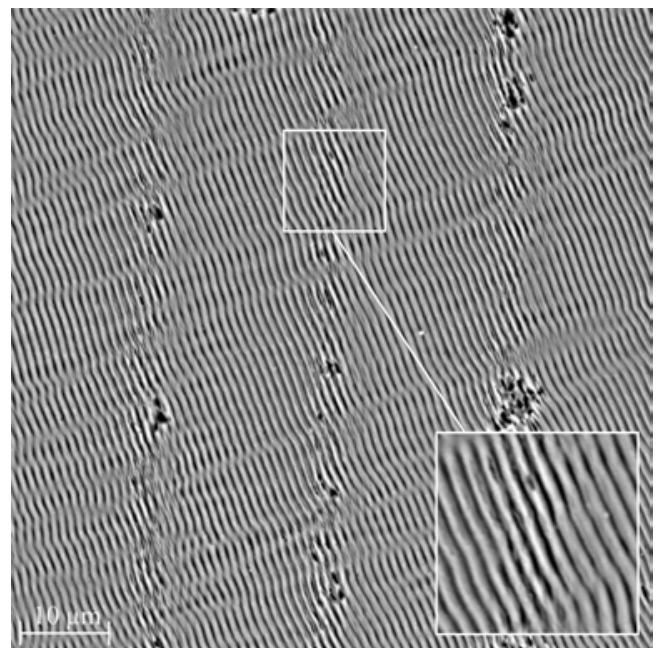

Fig. 7 SEM image of two-dimensional LSFL on sapphire generated with a scanning direction orthogonal to the laser polarization, $\mathrm{v}=80 \mathrm{~mm} / \mathrm{s}, \Phi=4.40 \mathrm{~J} / \mathrm{cm}^{2}$ and a line spacing of $20 \mu \mathrm{m}$.

\section{Conclusion}

We have demonstrated for the first time the generation of high quality two-dimensional low spatial frequency laser-induced periodic surface structures (2D-LSFL) on transparent material. By varying the laser fluence, scanning speed and scanning direction with respect to the polarization of the laser we have optimized the quality of 1D- and 2D-LSFL on sapphire in terms of homogeneity, parallelism and low damage. For structuring of entire surfaces with two-dimensional LSFL, a scanning strategy with a direction orthogonal to the laser polarization turns out to be preferential with laser fluence above the ablation threshold. These results may stimulate the application of 2D-LSFL on transparent materials in transmitted light microscopy and, more general, in medical applications by influencing wettability and cell growth.

\section{References}

[1] M. Birnbaum: J. Appl. Phys., 36, (1965) 3688.

[2] A. Cunha, A. P. Serro, V. Oliveira, A. Almeida, R. Vilar, M.-C. Durrieu: Appl. Surf. Sci., 265, (2013) 688.

[3] J. E. Sipe, J. F. Young, J. S. Preston, H. M. van Driel: Phys. Rev. B, 27, (1983) 1141.

[4] C. Albu, A. Dinescu, M. Filipescu, M. Ulmeanu, M. Zamfirescu: Appl. Surf. Sci., 278, (2013) 347.

[5] J. Bonse, A. Rosenfeld, J. Krüger: Appl. Phys., 106, (2009) 104910.

[6] Z. Fang, Y. Zhao, J. Shao: Optik, 127, (2016) 1171.

[7] D. C. Emmony, R. P. Howson, L. J. Willis: Appl. Phys. Lett., 23, (1973) 598.

[8] F. Liang, R. Vallée, S. L. Chin: Opt. Expess, 20, (2012) 4389.

[9] Q. Sun, F. Liang, R. Vallée, S. L. Chin: Opt. Lett., 33, (2008) 2713.

[10] R. Kuladeep, C. Sahoo, D. Narayana Rao: App. Phys. Lett., 104, (2014) 222103.

[11]P. Bizi-Bandoki, S. Benayoun, S. Valette, B. Beaugiraud, E. Audouard: Appl. Surf. Sci., 257, (2011) 5213

[12] A. Cunha, O. F. Zouani, L. Plawinski, A. M. B. Do Rego, A. Almeida, R. Vilar, M. C. Durrieu: Nanomed., 10, (2015) 725.

[13] K. Wallat, D. Dörr, R. Le Harzic, F. Stracke, D. Sauer, M. Neumeier, A. Kovtun, H. Zimmermann, M. Epple: J. Laser Appl., 24, (2012) 042016.

[14] S. Schwarz, S. Rung, R. Hellmann: Appl. Phys. Lett., 108, (2016) 181607.

[15] S. Höhm, A. Rosenfeld, J. Krüger, J. Bonse: J. Appl. Phys. 112 (2012), 014901.

[16]B. C. Stuart, M. D. Feit, A. M. Rubenchik, B. W. Shore, M. D. Perry: Phys. Rev. Lett., 74, (1995) 2248.

[17] R. Stoian, H. Varel, A. Rosenfeld, D. Ashkenasi, R. Kelly, E. E. B. Campbell: Appl. Surf. Sci., 165, (2000) 44.

[18]D. Ashkenasi, A. Rosenfeld, H. Varel, M. Wähmer, E. E. B. Campbell: Appl. Surf. Sci., 120, (1997) 65.

[19]L. Qi, K. Nishii, M. Yasui, H. Aoki, Y. Namba: Opt. Lasers Eng., 48, (2010) 1000.

[20] R. Wagner, J. Gottmann: J. Phys. Conf. Ser., 59, (2007) 333.

[21] S. Schwarz, S. Rung, R. Hellmann: Appl. Surf. Sci., 411 (2017) 113

[22] G. Eberle, M. Schmidt, F. Pude, K. Wegener: Appl. Surf. Sci., 378, (2016) 504.

[23] J. Gottmann, D. Wortmann, M. HörstmannJungemann: Appl. Surf. Sci., 255, (2009) 5641.

[24] J. Eichstädt, G. R. B. E. Römer, A. J. Huis in’t Veld: Phys. Procedia, 12, (2011) 7. 
[25] J. Bonse, R. Koter, M. Hartelt, D. Spaltmann, S. Pentzien, S. Höhm, A. Rosenfeld, J. Krüger: Appl. Phys. A, 117, (2014) 103.

[26] H. J. Scheel, T. Fukuda: "Crystal Growth Technology” (John Wiley \& Sons, 2003) p.499-501.

[27] M. Fox: “Optical Properties of Solids” (Oxford University Press, $\left.2^{\text {nd }}, 2010\right)$ p.9-10.

[28] J. Bonse, M. Munz, H. Sturm: J. Appl. Phys., 97, (2005) 013538.

[29] A. Borowiec, H. K. Haugen: Appl. Phys. Lett., 82, (2003) 4462.

[30] Y. Han, X. Zhao, S. Qu: Opt. Express, 19, (2011) 19150.

[31] J. M. Liu: Opt. Lett., 7, (1982) 196.

[32] C. Y. Zhang, J. W. Yao, C. Q. Li, Q. F. Dai, S. Lan, V. A. Trofimov, T. M. Lysak: Opt. Express, 21, (2013) 4439.

[33] J. Song, J. Ye, X. Lin, Y. Dai, G. Ma, H. Li, Y. Jiang, J. Qiu: Appl. Phys. A, 118 (2015) 1119.

[34] M. Barberoglou, D. Gray, E. Magoulakis, C. Fotakis, P. A. Loukakos, E. Stratakis: Opt. Express, 21, (2013) 18501.

[35] A. Ruiz de la Cruz, R. Lahoz, J. Siegel, G. F. de la Fuente, J. Soli: Opt. Lett., 8, (2014) 2491.

[36] S. Gräf, F. A. Müller: Appl. Surf. Sci., 331, (2015) 150.

[37]P. M. Fauchet, A. E. Siegman: Appl. Phys. Lett., 40, (1982) 824.

(Received: March 3, 2017, Accepted: May 15, 2017) 